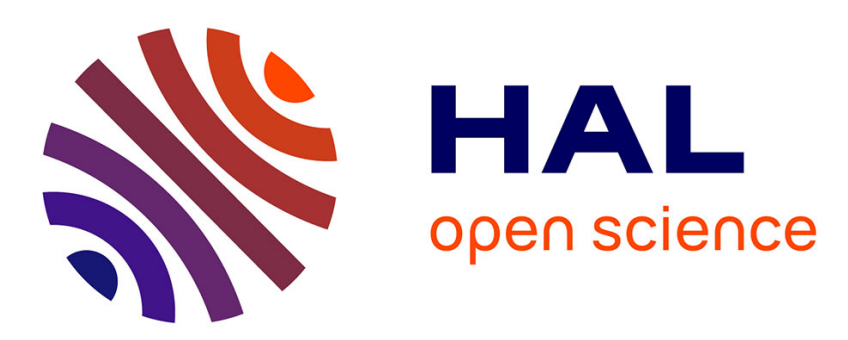

\title{
Buried Anti Resonant Reflecting Optical Waveguide based on porous silicon material for an integrated Mach Zehnder structure
}

Mohamed Hiraoui, Mohammed Guendouz, Nathalie Lorrain, Lazhar Haji, Mehrezi Oueslati

\section{To cite this version:}

Mohamed Hiraoui, Mohammed Guendouz, Nathalie Lorrain, Lazhar Haji, Mehrezi Oueslati. Buried Anti Resonant Reflecting Optical Waveguide based on porous silicon material for an integrated Mach Zehnder structure. Applied Physics Letters, 2012, 101 (19), pp.191114. 10.1063/1.4766729 . hal00804184

\section{HAL Id: hal-00804184 \\ https://hal.science/hal-00804184}

Submitted on 25 Mar 2013

HAL is a multi-disciplinary open access archive for the deposit and dissemination of scientific research documents, whether they are published or not. The documents may come from teaching and research institutions in France or abroad, or from public or private research centers.
L'archive ouverte pluridisciplinaire HAL, est destinée au dépôt et à la diffusion de documents scientifiques de niveau recherche, publiés ou non, émanant des établissements d'enseignement et de recherche français ou étrangers, des laboratoires publics ou privés. 


\title{
Buried Anti Resonant Reflecting Optical Waveguide
}

\section{based on porous silicon material for an integrated Mach Zehnder structure}

\author{
M. Hiraoui, ${ }^{1,2}$ M. Guendouz, ${ }^{1, a)}$, N. Lorrain, ${ }^{1}$ L. Haji ${ }^{1}$ and M. Oueslati ${ }^{2}$ \\ 1) Université Européenne de Bretagne, CNRS-UMR FOTON 6082, BP 80518 , \\ ENSSAT 6 rue Kerampont, 22305 Lannion Cedex, France \\ 2) Unité de Spectroscopie Raman, Faculté des Sciences de Tunis, 2092 El Manar- \\ Tunisia.
}

A buried Anti Resonant Reflecting Optical Waveguide for an integrated Mach Zehnder structure based on porous silicon material is achieved using a classical photolithography process. Three distinct porous silicon layers are then elaborated in a single step, by varying the porosity (thus the refractive index) and the thickness while respecting the anti-resonance conditions. Simulations and experimental results clearly show the antiresonant character of the buried waveguides. Significant variation of the reflectance and light propagation with different behavior depending on the polarization and the Mach Zehnder dimensions is obtained. Finally, we confirm the feasibility of this structure for sensing applications.

\footnotetext{
a) Electronic mail : mohammed.guendouz@univ-rennes1.fr
} 
Integrated optical devices are frequently used for communications ${ }^{1}$ and biochemical applications. ${ }^{2}$ In the last decade, optical interferometry ${ }^{3,4}$ is an evolving field which has been adopted for sensing applications. The Mach Zehnder (MZ) configuration appears to be the most widely used device in the field of biosensors thanks to its easy design and fabrication. ${ }^{5}$ Recently, MZ interferometers using various materials for chemical or biochemical sensing applications have been developed. ${ }^{6-9}$ In these MZ structures, the molecules to be detected are adsorbed at the surface of the interaction zone of the sensing arm and so will interact only with the evanescent field. ${ }^{10}$ Different kinds of resonant or antiresonant waveguides constituting the MZ structure can be constructed. 5,11

Porous silicon ${ }^{12}$ is an attractive and promising material which can be used in the fabrication of $\mathrm{MZ}$ interferometers for biosensing applications. The porosity and thus the refractive index can be easily varied as well as the thickness of the distinct porous layers of the structure. Moreover, the large internal specific area of the porous silicon layer is an asset in improving the sensitivity of the biosensor. Indeed, other porous structures have already been used to increase the sensitivity of waveguide based biosensors. ${ }^{13,14}$

Recently, we have demonstrated the feasibility of a planar AntiResonant Reflecting Optical Waveguide (ARROW) based on porous silicon for detection purposes even if the refractive index contrast is rather low ${ }^{15,16}$ compared to other materials. ${ }^{17,18}$

The particular characteristic of this antiresonant waveguide is that the upper porous layer performs both the role of active and guiding layer supporting field propagation which ensures a more sensitive interaction between the propagated light and the infiltrated biological species. Moreover, the realization of an integrated MZ structure requires a photolithography process 
which can be easily used with porous silicon material. Indeed, buried resonant waveguides based on porous silicon have been already demonstrated for telecommunication applications. ${ }^{19,20}$

In this work, we studied the integration of buried ARROW based on porous silicon in MZ structures used as optical transducers for biosensor applications. The propagation and modal characteristics of light in the waveguides and then in the MZ structure were investigated. We showed that the propagation of light was almost the same for both arms of the MZ structure. In order to realize a buried optical waveguide which is the main element of the interferometer MZ and to achieve a biosensor application, the structure must satisfy several conditions. These include the preparation of the porous layers with defined properties and the choice of the dimensions of the mask used. These parameters affect light propagation behavior with single or multi mode character and also the resonant or antiresonant effect.

The two arms of the MZ structures we fabricated consist of two buried waveguides, with lengths varying from 4 to $6 \mathrm{~mm}$, separated by a distance of $90 \mu \mathrm{m}$ and connected by $\mathrm{S}$ bends having a curvature radius of $5 \mathrm{~mm}$. The width of the waveguide apertures varies between 4 and $20 \mu \mathrm{m}$. The schematic and dimensions of the MZ structure are summarized in Fig. 1(a). The MZ structure was made using a photolithography process, performed on a heavily P doped (100) silicon substrate with a resistivity of $7 \mathrm{~m} \Omega . c m$, with a well defined mask designed using Olympios software. After photolithography step, an electrochemical anodization process was performed through the mask apertures to create three distinct porous silicon layers with porosities and thicknesses satisfying the antiresonance conditions ${ }^{21}$ to obtain the ARROW structures. ${ }^{15,16}$ For this anodization process, we used an electrolyte composed of HF (50\%), ethanol and deionised water (at $18 \mathrm{M} \Omega$ ) solution with ratios of 2-2-1 respectively. We applied successive current densities of 65,2 and $19 \mathrm{~mA} \cdot \mathrm{cm}^{-2}$ respectively for the layers. First, the core 
layer $\left(d_{c}\right)$ which has the lowest refractive index $\left(n_{c}=1.65 \pm 0.06\right)$ was created. Then the first cladding layer $\left(\mathrm{d}_{1}\right)$ with high refractive index $\left(\mathrm{n}_{1}=2.95 \pm 0.06\right)$ was made and this was followed by a second cladding layer $\left(\mathrm{d}_{2}\right)$ with a lower refractive index $\left(\mathrm{n}_{2}=2.07 \pm 0.06\right)$ than the previous one. The porosities and refractive indices are deduced from reflectance spectra using the Bruggeman model. ${ }^{22}$

SEM top view and cross section micrographs of the buried ARROW in the MZ structure with a width of $4 \mu \mathrm{m}$, are presented in Figs. 1(b) and 1(c) respectively. From the SEM top view we can clearly observe the two defined buried waveguides constituting the two arms of the MZ structure with a spacing of $90 \mu \mathrm{m}$. The cross section image of the waveguide clearly shows the three different porous silicon layers of the structure and their measured thicknesses. The waveguide appears as a half cylinder shape due to the anisotropy of the formation process related to the silicon crystallographic orientations. ${ }^{23,24}$

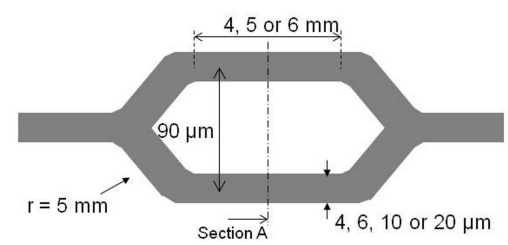

(a)

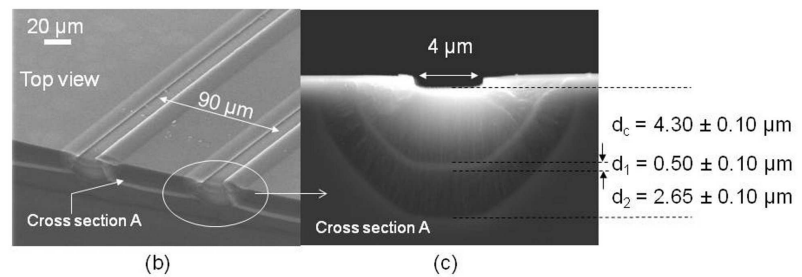

FIG. 1. Schematic and characteristic features of the MZ structure (a), SEM top view (b) and cross section (c) micrographs of the buried waveguides which compose the MZ structure. The width of the buried waveguide is $4 \mu \mathrm{m}$. 
In the case of an ARROW structure, unlike conventional waveguides, the light is confined in the core layer by total internal reflection at the upper air/core layer interface and by anti-resonant reflection due to the presence of the cladding layers underneath the core layer. The refractive index of the first cladding layer must be higher than those of the core and second cladding layer. The ARROW structure can therefore be considered as a leaky waveguide with radiation losses within the substrate reduced by the high reflection obtained by choosing the required thickness of the cladding layers. ${ }^{21}$

For a given thickness of the core layer $d_{c}$, the optimum thicknesses $\left(d_{1}\right.$ and $\left.d_{2}\right)$ of the two cladding layers for the $\mathrm{m}^{\text {th }}$ mode and for the $\mathrm{M}^{\text {th }}$ order of the antiresonant conditions must satisfy the following relationship ${ }^{21}$ :

$$
d_{1,2}=\frac{\lambda}{4 n_{1,2}}\left[1-\left(\frac{n_{c}}{n_{1,2}}\right)^{2}+\left(\frac{(m+1) \lambda}{2 n_{1,2} d_{c}}\right)^{2}\right]^{-1 / 2} .(2 M+1) \quad \mathrm{M}, \mathrm{m}=0,1,2, \ldots
$$

Where $\lambda(1.55 \mu \mathrm{m})$ is the light propagation wavelength along the $\mathrm{z}$ direction. In our case, we only have considered the fundamental mode $(\mathrm{m}=0)$ which corresponds to a single mode propagation.

The reflectivity $(\mathrm{R})$ at the interface between the core and the first cladding layer is a key parameter for an ARROW structure. The reflectivity expression for any polarization (TE or TM) is obtained by calculations based on the Fresnel coefficients. ${ }^{25}$ Using these, $\mathrm{R}$ was calculated ${ }^{16}$ and plotted in Fig. 2 for both TE and TM polarizations as a function of $\mathrm{d}_{1}$ for $\mathrm{d}_{\mathrm{c}}=4.3 \mu \mathrm{m}$ and at a working wavelength of $\lambda=1.55 \mu \mathrm{m}$. 


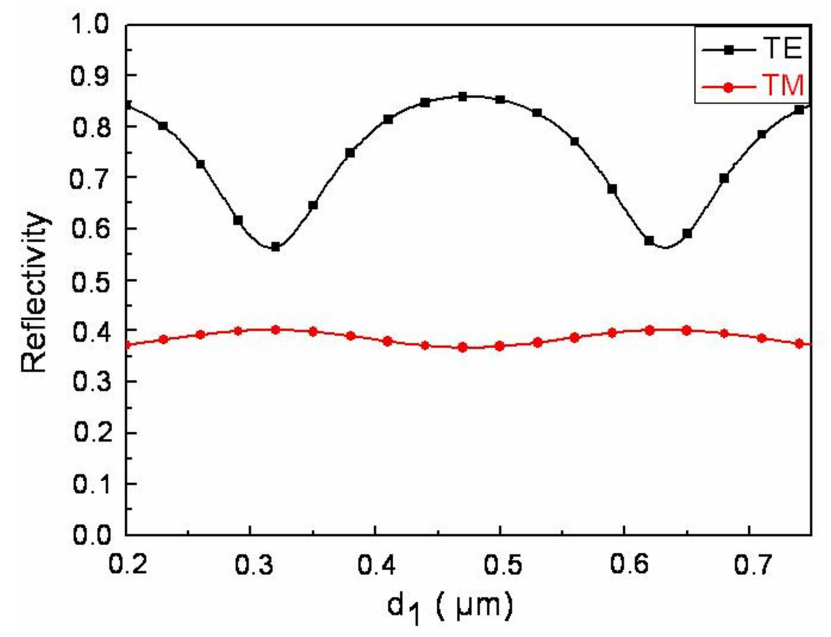

FIG. 2. Calculated reflectivity as a function of $\mathrm{d}_{1}$ for $\mathrm{d}_{\mathrm{c}}=4.3 \mu \mathrm{m}$ for both TE and TM polarizations, with fixed experimental refractive index values $n_{c}=1.65 \pm 0.06, n_{1}=2.95 \pm 0.06$, $\mathrm{n}_{2}=2.07 \pm 0.06$ and at a working wavelength of $\lambda=1.55 \mu \mathrm{m}$.

From this figure, we see that the maximum reflectivity $\left(\mathrm{R}_{\max } \approx 0.85\right)$ for the TE mode is obtained for values of $d_{1}$ around $0.5 \mu \mathrm{m}$ corresponding to the first order of the anti-resonance conditions. In the case of the TM mode, we note that the reflectivity is low and also that the difference between the maximum and minimum of reflectivity is lower than for the TE mode. This distinction in the reflectivity between the TE and TM modes clearly reflects the feature of the ARROW structure. ${ }^{21}$ The experimental thickness of the first cladding layer measured by SEM was about $0.5 \mu \mathrm{m}$ (Fig. 1(c)), a value which corresponds to a maximum of the reflectivity according to Fig. 2. Thus, the antiresonant conditions are respected in the buried waveguides constituting the MZ structure.

Furthermore, the Beam Propagation Method (BPM) ${ }^{26}$ was used to simulate the field evolution along the propagation direction $(\mathrm{z})$ in the waveguide taking into account the 
experimental values of the refractive index and thickness. In Fig. 3, we present the cross section images of the light propagated along the $\mathrm{z}$ direction of the $10 \mathrm{~mm}$ length ARROW structure for both TE (Figs. 3(a) and 3(c)) and TM (Fig. 3(b) and 3(d)) polarizations. Also, the profiles of the field measured in the vertical direction at the input (start field) and at the output (end field) of the waveguide are reported. The estimated boundaries of the three layers are indicated by the dashed lines.

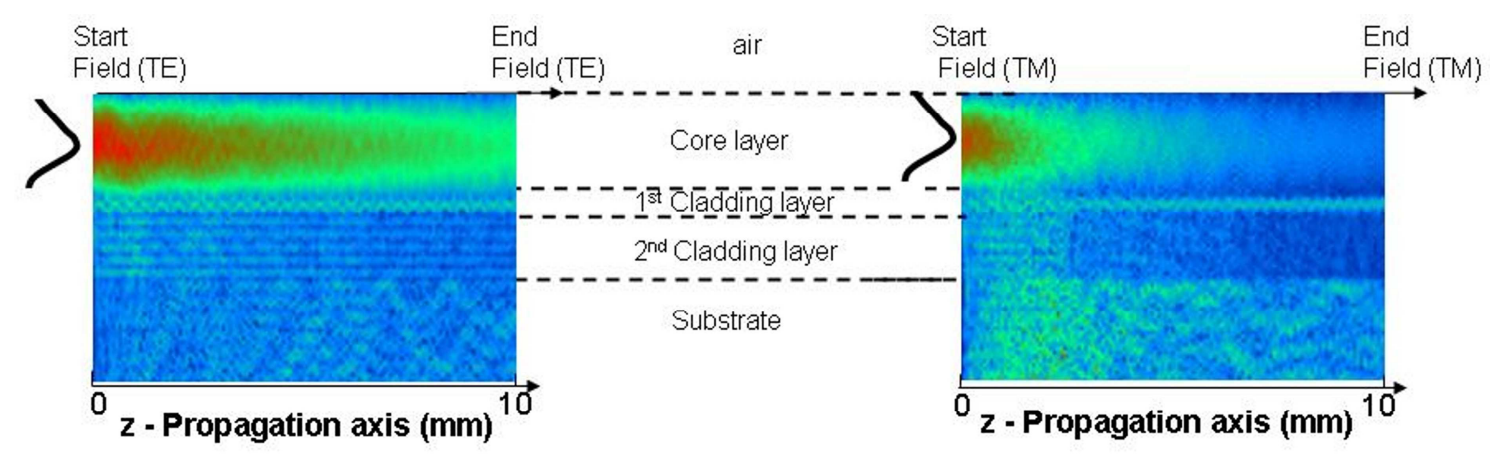

(a) TE Mode

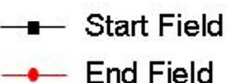

(b) TM Mode

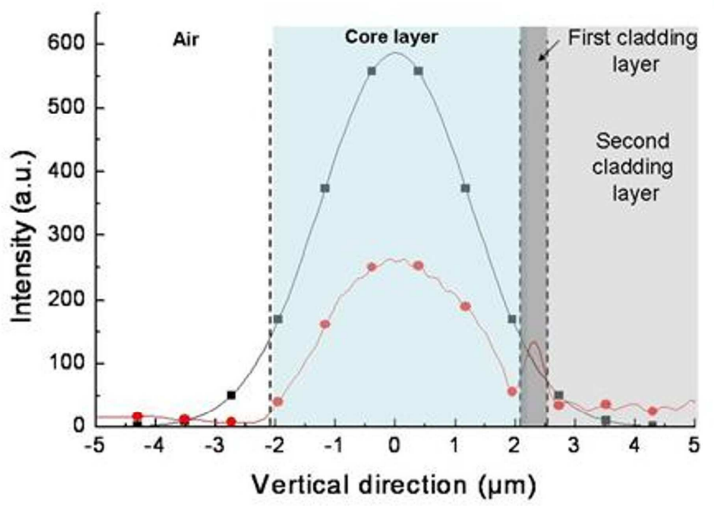

(c) TE Mode

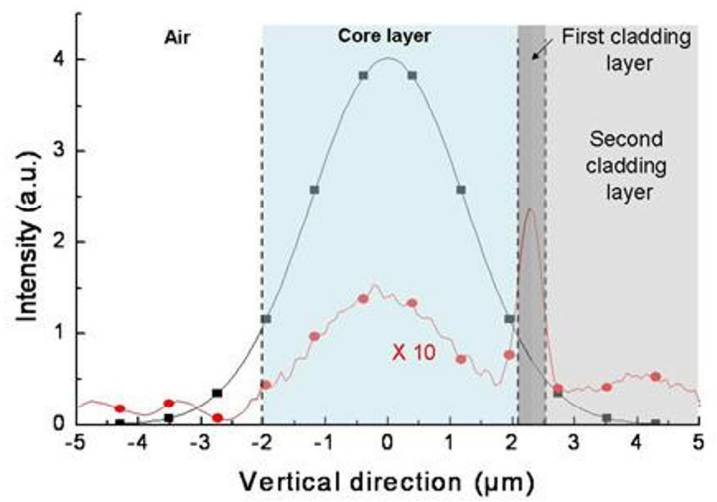

(d) TM Mode

FIG. 3. Cross section images of the light propagated along the $\mathrm{z}$ direction and simulated near field profiles of the ARROW structure for TE $(a, c)$ and TM $(b, d)$ polarizations with the following parameters: refractive index values $\mathrm{n}_{\mathrm{c}}=1.65, \mathrm{n}_{1}=2.95, \mathrm{n}_{2}=2.07$, a working wavelength $\lambda=1.55 \mu \mathrm{m}$, and thickness values $d_{c}=4.3 \mu \mathrm{m}, d_{1}=0.5 \mu \mathrm{m}$. 
The simulated profiles indicate that the light propagated in the core of the waveguide is reduced in intensity, compared to the input light, after a propagation of $10 \mathrm{~mm}$ for the TE mode and even more so for the TM mode. The estimated optical losses, calculated from the integrated area under each propagated profile at the input and at the output of the waveguide (Fig. 3), are equal to $4 \pm 1 \mathrm{~dB} / \mathrm{cm}$ and $13 \pm 1 \mathrm{~dB} / \mathrm{cm}$ respectively for TE and TM modes for the waveguide width of $4 \mu \mathrm{m}$. Moreover, in the case of the TE mode, the propagated optical field is located mainly in the core layer despite its low refractive index. However, for the TM mode, the light is mostly guided in the first cladding layer. This can be explained by the fact that the TM mode has a lower reflection coefficient compared to the TE one (Fig. 2) and that the refractive index contrast between the core and the first cladding layers is rather high.

In order to experimentally verify the antiresonant feature of the buried waveguides constituting the MZ structure, we carried out near-field measurements to check the changes in the modal characteristics of the propagated light in the MZ structure for both polarizations. Figures 4(a) and 4(b) illustrate the near field profiles of the light at the MZ structure output for both polarizations and for widths equal to $4 \mu \mathrm{m}$ and $20 \mu \mathrm{m}$ respectively. In Fig. 4(a) (solid line), we see that, in the case of TE mode, the light is guided almost completely in the core layer which has the lowest refractive index. On the other hand, for the TM mode (dashed line), the light is propagated principally in the first cladding layer which has the highest refractive index and with only a small amount of light propagated in the core layer. Furthermore, in Fig. 4(b), it can be noticed that near field profiles present a very similar behavior but with less confinement of light due to the increase of the width waveguide $(20 \mu \mathrm{m})$. Therefore, the buried waveguides constituting the $\mathrm{MZ}$ structure are seen to respect the antiresonant feature and all these results are in good agreement with the ones given by BPM simulation. 

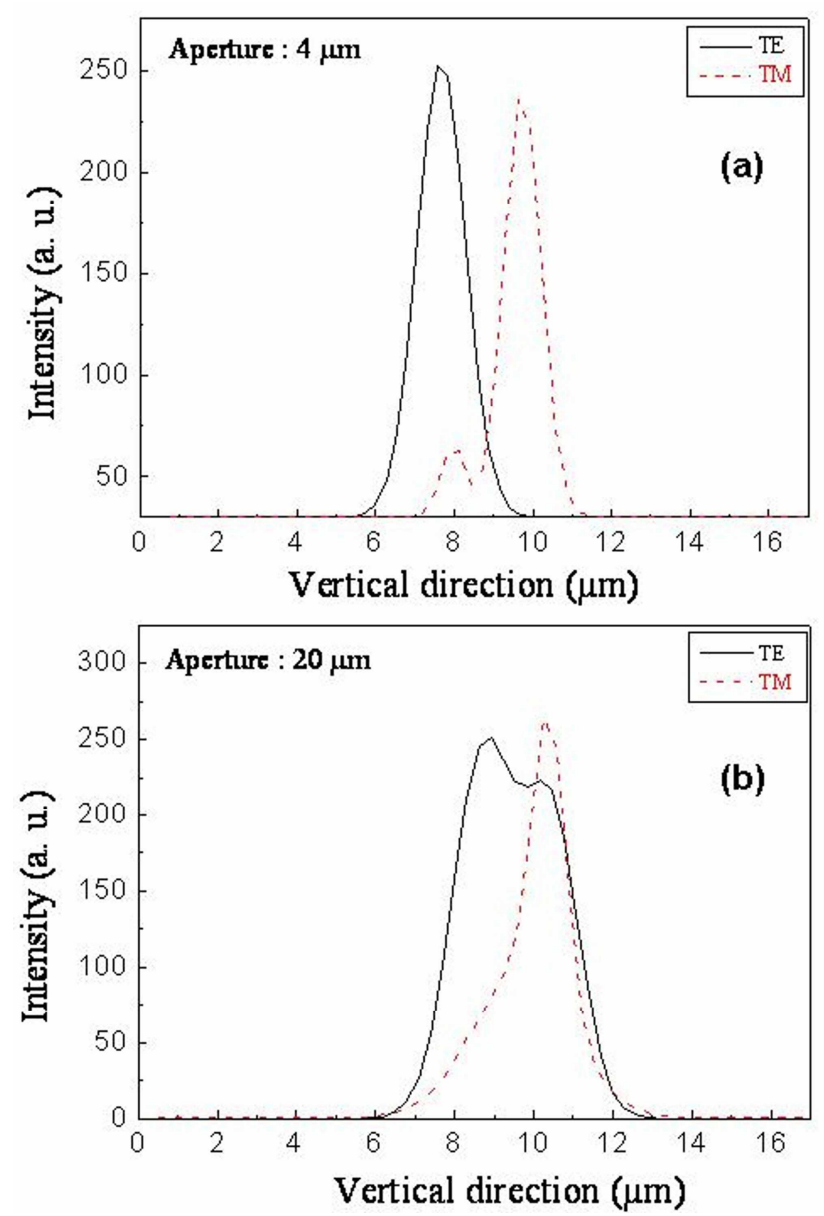

FIG. 4. Experimental near-field profiles for both TE and TM polarizations at the MZ structure output and for waveguide widths of $4 \mu \mathrm{m}$ (a) and $20 \mu \mathrm{m}$ (b).

We have also cleaved the MZ structure in the middle (Fig. 5(a)) in order to verify the light propagation in the two arms. The near-field images measured and the 3D intensity profiles for the width of $4 \mu \mathrm{m}$ (Fig. 5(b) and 5(c)) at the output of each arm of the MZ structure are also indicated. The intensity of the light is almost the same and the field distribution corresponds to a single mode feature. Indeed, the SEM cross section micrograph (Fig. 1(c)) of the buried 
waveguide constituting the $\mathrm{MZ}$ structure clearly presents, for the width of $4 \mu \mathrm{m}$, a shape that allows good light confinement in the core layer both in the horizontal and vertical directions.

We have also verified the light propagation for the TM polarization which indicates the same behavior as in the case of TE polarization but with a change in the output light intensity. This demonstrates that we have developed a symmetrical MZ structure made with two similar single mode buried ARROW for an aperture of $4 \mu \mathrm{m}$. This is an advantage for biosensing applications since a good contrast of the interference fringes between the reference arm and the sensing arm would be obtained. ${ }^{5}$

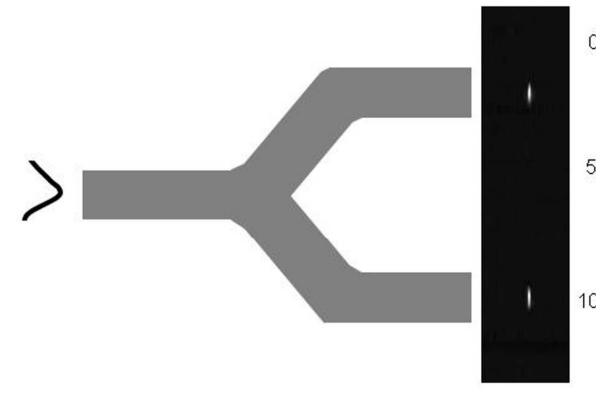

(a) (b)

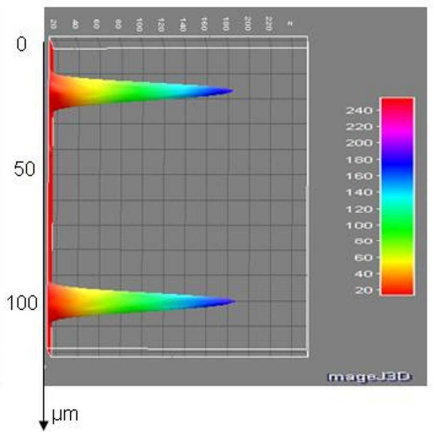

(c)

FIG. 5. Schematic of the cleaved MZ structure (a). Image of the near-field profiles at the output of each arm of the cleaved MZ for a width of $4 \mu \mathrm{m}(\mathrm{b})$ and corresponding 3D intensity profiles (c). The input signal power was $3 \mathrm{~mW}$.

Our interest in this work is to use the buried ARROW-MZ structure as an optical transducer for biosensing applications by measuring the phase change between the reference arm and the active one after the bio-functionalization steps. 
Since porous silicon material is strongly hydrophobic and unstable, a partial or total oxidation of the porous structure is necessary, depending on the intended application such as biosensing where a functionalization process is needed. ${ }^{27}$ Consequently, in order to use the MZ structure based on porous silicon as a sensor, a second photolithography step to create the aperture in the detection arm through which the molecules will be infiltrated, is required. This is carried out after a heat treatment in wet oxygen. The sensor has been tested with ethanol to prove the feasibility of this kind of MZ structure for sensing applications.

Figure 6 shows the evolution of the intensity, as measured by the photodiode, of the MZ output signal versus time for an interaction length $\mathrm{L}=5 \mathrm{~mm}$. As soon as the ethanol is introduced using a micro-syringe, we notice the appearance of interference fringes whose amplitude varies significantly. Consequently, the uncertainty in the number of fringes recorded is quite high due to optical losses and ethanol evaporation. ${ }^{28}$ By counting the number of fringes $(\mathrm{p}=16 \pm 2)$ and considering complete ethanol evaporation, we deduce a refractive index variation $\Delta n=2.48 \pm 0.3110^{-3}$ in the interaction area of the sensing arm using the following relationship: $\Delta n=\frac{p \lambda}{L}$ where $\lambda$ is the working wavelength $(\lambda=1.55 \mu \mathrm{m})$. This preliminary result is encouraging, but further study is needed to improve the performance of this interferometric structure based on porous silicon for sensing applications. 


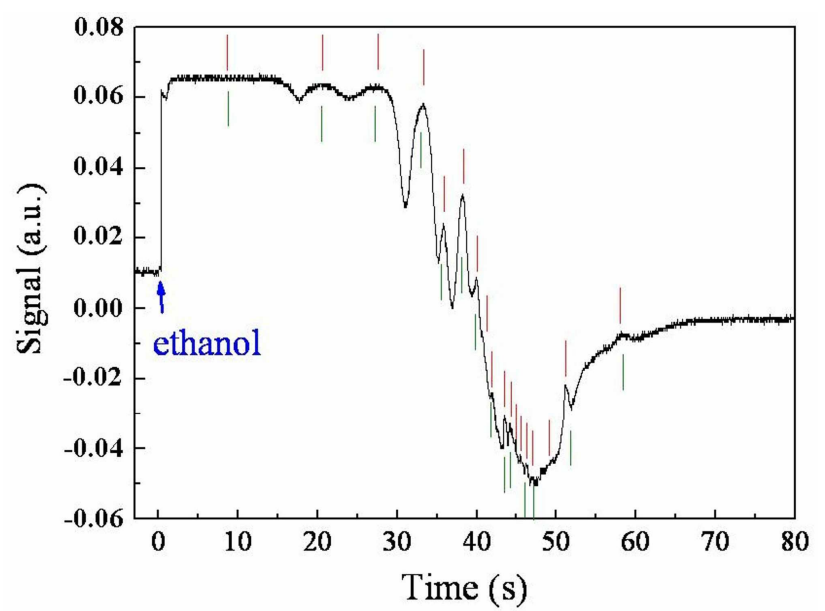

FIG. 6. Sensor response for a change of the refractive index in the detection arm using ethanol.

The design, fabrication and optical study of buried waveguide integrated in a MZ structure based on porous silicon material have been presented. The theoretical study of reflectance and BPM simulation allowed us to predict the propagated field along the propagation direction in the structure, taking into account the experimental values of the refractive index and of the thickness satisfying the antiresonant conditions for both polarizations. Optical near-field measurements were carried out in order to check the light propagation behavior along the $\mathrm{MZ}$ structure. The experimental results have indicated that for the TE mode, the optical propagated field is located mainly in the core layer despite its low refractive index whereas for the TM mode, the light is mostly guided in the first cladding layer. Therefore, both theoretical and experimental studies, carried out without any post-treatment, demonstrate that the buried waveguides constituting the MZ structure satisfy the antiresonant condition. These preliminary studies have proved the feasibility of fabricating a MZ structure with buried ARROW based on porous silicon. In addition, testing of the $\mathrm{MZ}$ with ethanol has shown that such a structure can be 
used for sensing applications. Our current work, in progress, is to test other biomolecules such as BSA and HSA proteins using this integrated MZ structure.

\section{Acknowledgments}

This work is supported by the program PHC-Utique administered by the Joint Committee of University Cooperation (CMCU), $\mathrm{N}^{\circ} 11 \mathrm{G} 1109$. 


\section{References}

${ }^{1}$ R. C. Alferness, IEEE J. Quantum Electron. 17, 946 (1981).

${ }^{2}$ P.V. Lambeck, Sens. Actuators B. 8, 103 (1992).

${ }^{3}$ R. Liu, T.A. Schmedake, Y.Y. Li, M.J. Sailor, Y. Fainman, Sens. Actuators B. 87, 58 (2002).

${ }^{4}$ P.L. Edmiston, D.P. Campbell, D.S. Gottfried, J. Baughman, M. M. Timmers, Sens. Actuators

B. 143, 574 (2010).

${ }^{5}$ E. F. Schipper, A. M. Brugman, C. Dominguez, L. M Lechuga, R. P.H. Kooyman, and J. Greve, Sens. Actuators B. 40, 147 (1997).

${ }^{6}$ R.G. Heideman 1, P.V. Lambeck, Sens. Actuators B. 61, 100 (1999).

${ }^{7}$ F. Prieto, B. Sepulveda, A. Calle, A. Llobera, C. Dominguez, and L.M. Lechuga, Sens. Actuators B. 92, 151 (2003).

${ }^{8}$ B. Maisenhölder, H. P. Zappe, R. E. Kunz, P. Riel, M. Moser, J. Edlinger, Sens. Actuators B. 38-39, 324 (1997).

${ }^{9}$ P. Kozma, A. Hamori, S. Kurunczi, K. Cottier, R. Horvath, Sens. Actuators B. 155, 446 (2003).

${ }^{10}$ B. Drapp, J. Piehler, A. Brecht, G. Gauglitz, B.J. Luff, J.S. Wilkinson and J. Ingenhoff, Sens. Actuators B. 38, 277 (1997).

${ }^{11}$ D. Jimenez, E. Bartolome, M. Moreno, J. Munoz and C. Dominguez, Optics Communications 132, 437 (1996).

${ }^{12}$ A. Halimaoui, Surface Science Letters. 306, 550 (1994).

${ }^{13}$ R. Horvath, H. C. Pedersen and N. B. Larsen, Appl. Phys. Lett. 81, 2166 (2002).

${ }^{14}$ R. Horvath, K. Cottier, H. C. Pedersen and J. J. Ramsden, Biosensors and Bioelectronics. 24, 799 (2008).

${ }^{15}$ L. Haji, M. Hiraoui, N. Lorrain and M. Guendouz, Appl. Phys. Lett. 100, 111102 (2012).

${ }^{16}$ M. Hiraoui, L. Haji, M. Guendouz, N. Lorrain, A. Moadhen and M. Oueslati, Biosensors and Bioelectronics 36, 212 (2012).

${ }^{17}$ F. Prieto, L. M. Lechuga, A. Calle, A. Llobera, C. Dominguez, Journal of Lightwave Technology. 19, 75 (2001).

${ }^{18}$ Shruti, R.K. Sinha, R. Bhattacharya, Opt. Quant. Electron. 41, 181 (2009).

${ }^{19}$ J. Charrier, C. Lupi, L. Haji, C. Boisrobert, Materials Science in Semiconductor Processing 3, 357 (2000). 
${ }^{20}$ V. P. Bondarenko, A. M. Dorofeev and N. M. Kazwchits, Microelectronic Engineering. 28, 447 (1995).

${ }^{21}$ M. A. Duguay, Y. Kokubun and T.L. Koch, Appl. Phys. Lett. 49, 13 (1986).

${ }^{22}$ D. E Aspnes, Thin Solid Films 89, 249 (1982).

${ }^{23}$ M. Guendouz, P. Joubert and M. Sarret, Materials Science and Engineering B. 69, 43 (2000).

${ }^{24}$ S. Nagata, S. Matsushita, K. Saito, Y. Ohshita, Y. Maeda, M. Yamaguchi and A. J. Ikushima, Appl. Phys. Lett. 82, 16 (2003).

${ }^{25}$ M. Brinkmann, J. Hayden, M. Letz, Springer handbook of lasers and optics, ed. F. Trager New York (2007).

${ }^{26}$ J. Yamauchi, J. Shibayama, O. Saito, O. Uchiyama and H. Nakano, J. Lightwave Technol. 14, 2401 (1996).

${ }^{27}$ M.I. Baraton, W. Chang and B.H. Kear, J. Phys. Chem. 100, 16647 (1996).

${ }^{28}$ Th. Schubert, N. Haase, H. Huck, R. Gottfried, Sensors and Actuators Physical. 60, 108 (1997). 


\section{List of figure Captions:}

FIG. 1. Schematic and characteristic features of the MZ structure (a), SEM top view (b) and cross section (c) micrographs of the buried waveguide which compose the MZ structure. The width of the buried waveguide is $4 \mu \mathrm{m}$.

FIG. 2. Calculated reflectivity as a function of $d_{1}$ for $d_{c}=4.3 \mu \mathrm{m}$ for both TE and TM polarizations, with fixed experimental refractive index values $n_{c}=1.65 \pm 0.06, n_{1}=2.95 \pm 0.06$, $\mathrm{n}_{2}=2.07 \pm 0.06$ and at working wavelength $\lambda=1.55 \mu \mathrm{m}$.

FIG. 3. Cross section images and simulated near field profiles of the ARROW structure obtained using the Beam Propagation Method (BPM) for TE (a, c) and TM (b, d) polarizations with the following parameters: refractive index values $\mathrm{n}_{\mathrm{c}}=1.65, \mathrm{n}_{1}=2.95, \mathrm{n}_{2}=2.07$, a working wavelength $\lambda=1.55 \mu \mathrm{m}$, and thickness values $\mathrm{d}_{\mathrm{c}}=4.3 \mu \mathrm{m}, \mathrm{d}_{1}=0.5 \mu \mathrm{m}$.

FIG. 4. Experimental near-field profiles for both TE and TM polarizations at the MZ structure output and for waveguide widths of $4 \mu \mathrm{m}$ (a) and $20 \mu \mathrm{m}$ (b).

FIG. 5. Schematic of the cleaved MZ structure (a). Image of the near-field profiles at the output of each arm of the realized cleaved $\mathrm{MZ}$ for a width of $4 \mu \mathrm{m}$ (b) and corresponding 3D intensity profiles (c). The power of the input signal is $3 \mathrm{~mW}$.

FIG. 6. Sensor response for a change of the refractive index in the detection arm using ethanol solution. 